\title{
General relativity in upper secondary school: Design and evaluation of an online learning environment using the model of educational reconstruction
}

\author{
Magdalena Kersting," Ellen Karoline Henriksen, Maria Vetleseter Bøe, and Carl Angell \\ Department of Physics, University of Oslo, P.O. Box 1048 Blindern, 0316 Oslo, Norway
}

(Received 27 September 2017; revised manuscript received 3 April 2018; published 25 May 2018)

\begin{abstract}
Because of its abstract nature, Albert Einstein's theory of general relativity is rarely present in school physics curricula. Although the educational community has started to investigate ways of bringing general relativity to classrooms, field-tested educational material is rare. Employing the model of educational reconstruction, we present a collaborative online learning environment that was introduced to final year students (18-19 years old) in six Norwegian upper secondary physics classrooms. Design-based research methods guided the development of the learning resources, which were based on a sociocultural view of learning and a historical-philosophical approach to teaching general relativity. To characterize students' learning from and interaction with the learning environment we analyzed focus group interviews and students' oral and written responses to assigned problems and discussion tasks. Our findings show how design choices on different levels can support or hinder understanding of general relativity, leading to the formulation of design principles that help to foster qualitative understanding and encourage collaborative learning. The results indicate that upper secondary students can obtain a qualitative understanding of general relativity when provided with appropriately designed learning resources and sufficient scaffolding of learning through interaction with teacher and peers.
\end{abstract}

DOI: 10.1103/PhysRevPhysEducRes.14.010130

\section{INTRODUCTION}

Albert Einstein's theory of general relativity is an important pillar of modern physics. Classically, gravity is described as a force, but Einstein described gravitational effects as a manifestation of a deep connection between time, space, and massive objects. This revolutionary insight is rarely present in school physics curricula because general relativity (GR) is abstract in a double sense: First, learners lack experience with relativistic phenomena, because the realm of GR covers extreme situations not to be found in everyday life. Second, GR builds on a sophisticated mathematical framework that is not readily accessible to secondary school students.

Nonetheless, physicists and science educators argue for introducing GR in schools because of its far-reaching scientific, philosophical, and cultural importance [1-3]. Not only does the theory constitute our best understanding of the Universe, it continues to inspire scientific and technological progress and has become an issue of practical concern to mainstream physicists and engineers [4]. GPS

\footnotetext{
*magdalena.kersting@fys.uio.no
}

Published by the American Physical Society under the terms of the Creative Commons Attribution 4.0 International license. Further distribution of this work must maintain attribution to the author(s) and the published article's title, journal citation, and DOI. technology relies on GR as does the discovery of gravitational waves that caught public interest in 2016 [5].

Clearly, when introducing GR to school physics, we need to find ways of communicating important features of GR without relying on its advanced mathematical foundations [6]. However, it seems that the educational community has not properly laid the groundwork for bringing GR to schools [7]. Studies on secondary school students' conceptual development of key concepts in GR are scarce. Most research papers focus either on special instead of general relativity [8-10] or investigate learning among undergraduate university students [4,11-13]. Relying mostly on case studies and interviews, these studies agree in their findings that students struggle with the interpretation of relativistic phenomena and that they hold a large number of misconceptions.

Despite first steps towards teaching GR in schools and an increasing awareness to teach GR at the undergraduate level [4], there is a lack of research literature describing field-tested learning resources in GR that are tailored to the needs of secondary school students. The present paper aims to fill this gap by reporting on the development and evaluation of an online learning environment and upper secondary students' understanding of key concepts in GR.

\section{BACKGROUND OF THE STUDY}

In this section we present the greater educational project that this work is part of and discuss the curriculum 
constraints of learning physics in Norway to contextualize our study and its results.

\section{A. Project ReleQuant}

This study was conducted within the broader ReleQuant project that develops collaborative online learning environments in general relativity and quantum physics for the optional physics subject in the final year of upper secondary school in Norway [14]. ReleQuant was established to investigate novel ways of teaching modern physics and to study students' learning processes and motivation. In the domain of modern physics, demands on both students and teachers are high. Students struggle with relativity and quantum physics, since these topics describe phenomena that cannot be visualized or experienced directly $[7,15,16]$. Often, teachers lack sufficient background in modern physics, thus finding the topics conceptually demanding [17]. Moreover, by emphasizing qualitative understanding and philosophical aspects, the Norwegian curriculum requires novel ways of teaching and learning that differ from teacher-centered traditional physics lessons focused on content knowledge and mathematical problem solving.

The development of learning resources in ReleQuant is based on a sociocultural perspective on learning, which emphasizes language as an integral part of the learning process $[18,19]$. This approach entails that students make physics concepts their own through use of language and interaction with others $[19,20]$. In particular in the domain of GR, upper secondary students have to rely on language since the advanced mathematical framework of GR is not available.

Another feature of the development of ReleQuant resources is the inclusion of history, philosophy, and nature of science (NOS) aspects. History, philosophy, and NOS have long been advocated as important elements in science education [21,22]. Physics educators have made calls for physics education to move beyond traditional contentfocused instruction and include historical, epistemological, and sociocultural aspects (see, e.g., Ref. [23]). Moreover, research has shown that use of history, philosophy, and NOS can foster understanding in relativity and quantum physics content [8,24], making this a suitable approach for an educational reconstruction of GR that focuses on qualitative understanding.

\section{B. General relativity in the physics curriculum}

With the school reform introduced in autumn 2006, GR was included in the Norwegian curriculum for upper secondary physics. Such a physics curriculum is uncommon. Of five other European countries and Australia, which have an educational system comparable to Norway's, only Sweden includes GR [15]. In addition to the more formalistic and traditional topics on the curriculum, such as Newtonian mechanics and electromagnetism, Norwegian physics students are expected to explore different interpretations and philosophical aspects of modern physics. However, the specific curricular goal for GR remains vague:

The aims of the studies are to enable pupils to give an account of the postulations that form the basis for the special theory of relativity, discuss qualitatively some of the consequences of this theory for time, momentum and energy, and give a qualitative description of the general theory of relativity [25].

This broad description leaves scope for various interpretations of what constitutes GR qualitatively. Indeed, the physics curriculum in Norway gives some guidance for interpretation since it emphasizes historical and philosophical aspects of physics in particular:

[The subject] also deals with how scientific knowledge is established and with conflicts and dilemmas that might arise during this process. (...) [the] subject shall help create an awareness that physics is part of our cultural heritage and that the subject must be viewed in a historical perspective [25].

In addition, the curriculum emphasizes basic skills such as expressing oneself orally and in writing when learning physics. Thus, the approach chosen in the present work aligns both with the Norwegian curriculum goals and with research literature that describes the important role of language in a sociocultural setting $[19,20]$, and that recommends to include historical and philosophical aspects in school science in order to foster understanding of the nature of science [21]. We believe that such an approach will be broadly applicable to preuniversity physics curricula in other countries as well, thus advancing modern physics education in general.

\section{THEORETICAL AND METHODOLOGICAL FRAMEWORK}

In this study, we combine two powerful frameworks: The model of educational reconstruction (MER) [26] provides a theoretical frame for choices of physics content and learning resources, while design-based research (DBR) [27] serves as a methodological frame for the development and evaluation of the learning resources.

Both DBR and MER draw on the tradition of designing and evaluating teaching-learning sequences (TLS). In a special issue of the International Journal of Science Education on TLSs, Méheut and Psillos note in the editorial:

Yet whereas there is often extensive communication of learning results, the various explicit and implicit assumptions and decisions concerning the design of a TLS, its teaching features or the interlacing of teaching with 
learning are less widely discussed, and may not even be made clear and comprehensible [28].

By dividing the design process into distinct phases, MER and DBR address this challenge and offer methods for validating and reporting on the design of learning environments.

\section{A. Model of educational reconstruction}

The model of educational reconstruction [26] offers a methodological framework for science education research and provides guidance for integrating empirical research on teaching and learning in the development of content structures for instruction and learning resources [28,29]. The framework comprises the basic idea that three strands of educational research are closely connected (Fig. 1): First, to analyze the particular science content of a topic and to identify its key concepts. Second, to take student and teacher perspectives into account and to assess the crucial features of students' learning processes in this topic. Third, to design and evaluate learning environments and suitable learning activities.

MER aligns well with the objectives of this study since its broad and holistic approach can serve as a useful tool for scrutinizing the educational relevance of fields of science that have not entered mainstream education yet. In particular in the field of physics, the model has previously been employed to reconstruct novel learning domains such as chaos theory [30], nonlinear systems [31,32], nanoscience [33,34], climate change [35], and physical geography [36].

In this study, an analysis of the science content structure of GR and students' perspectives according to the literature served as a basis for the design, development, and evaluation of an online learning environment in GR. Presenting new empirical results, we add, moreover, to the understanding of upper secondary physics students' learning challenges and discuss the mutual relation between the three strands of MER.

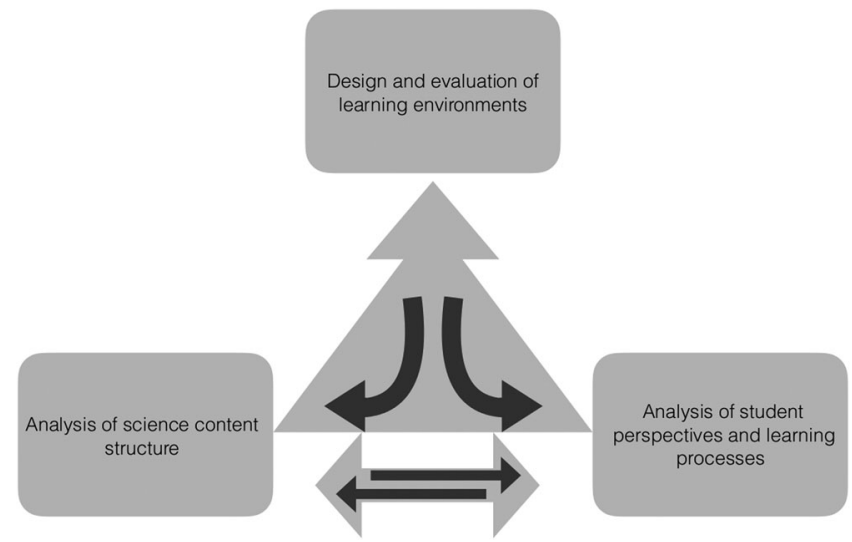

FIG. 1. The model of educational reconstruction combines three strands of educational research iteratively [26].

\section{B. Design-based research}

The main goal of the DBR methodology, also referred to as educational design research [37], is to develop and implement systematic solutions to educational problems $[27,38]$. The framework offers suitable tools for developing field-tested learning resources, because it relies on repeated rounds of development in close collaboration with practitioners. The iterative phases comprise analysis and reflection of the problem, development and design of solutions, and evaluation using classroom research [37]. Bridging the gap from research to classroom settings, the formulation of design principles is a common result of DBR research praxis $[17,39]$.

Focusing on design revisions and reporting more generally on the design research and its elements, goals, and phases are characteristic features of DBR methods [40]. Therefore, we have made the design of our learning environment a crucial part of our research agenda. In this study, tentative design hypotheses were derived from the content structure for instruction that guided the development of the learning environment. Testing these design hypotheses in the classroom setting allowed for iterative revisions in the design process and enabled us to formulate research-based design principles.

In line with the stance of project ReleQuant, the design of learning resources drew on the sociocultural tradition of viewing knowledge as constructed within and distributed among people and their environments [41]. In particular, we view learning and conceptual development in GR as a process of students' interaction with peers and the teacher and with their (physical and technological) environment. Students in the physics classroom construct knowledge through collaborative activities that are partly mediated through the online learning environment. In line with Vygotsky [42], who highlights the interrelationship of language and the development of abstract thoughts, our teaching approach uses discussion and writing tasks as cognitive tools. "Talking physics" $[19,43]$ is one important way to reach understanding of abstract relativistic concepts.

\section{Aims and research questions}

The aim of this study is to describe and propose an educational reconstruction of GR based on the science content structure and published literature on teaching and learning the topic supplemented with our own empirical results. Specifically, we have investigated Norwegian upper secondary physics students' work and interaction with an online learning environment to answer the following research questions:

- RQ1: What characterizes the understanding of key features of GR that participating students express while engaging with the learning environment?

- RQ2: In what ways do the participating students' experiences support or challenge the design hypotheses 
that guided the development of our online learning environment?

- RQ3: Based on findings from RQ1 and RQ2, what design principles can be formulated for the development of learning resources in GR at the upper secondary level?

\section{AN EDUCATIONAL RECONSTRUCTION OF GENERAL RELATIVITY}

Following the organization of MER, in this section we bring together all three strands that have guided our attempt to reconstruct GR from an educational perspective. The first steps towards an educational reconstruction of GR consisted of identifying key concepts of GR and summarizing published research concerning students' conceptions and challenges. Based on these insights, we created a content structure for instruction consisting of a sequence of specific learning goals and design hypotheses that provided guidance for the development of the learning environment. We then tested the learning environment in classrooms and evaluated and adjusted the design iteratively. In the subsequent sections, we will report on the 2016 class room trial of this learning environment and the resulting design principles.

\section{A. MER-component 1: Key concepts of general relativity according to textbooks}

To identify key concepts of GR and to gain insight into how educators treat those concepts, we started by analyzing the presentation of GR in the two Norwegian upper secondary physics textbooks that are on the market $[44,45]$. This analysis enabled an insight into how the book authors interpreted the vague curriculum goals explicitly. Since the Austrian physics curriculum treats GR similarly to the Norwegian one [46], an Austrian textbook [47] was scrutinized to obtain an additional and possibly contrasting educational perspective.

All three books present similar key features of GR and differ mostly in the degree of given details. The principle of equivalence and its implication to the equality of gravitational and inertial mass serves as a first step into the topic: Locally, one cannot distinguish between a gravitational field and uniform acceleration. This is followed by a discussion of reference frames and a generalized version of the principle of relativity: An inertial frame is a reference frame in free fall and the laws of physics take the same form in all reference frames. Gravitational redshift, gravitational bending of light, and gravitational time dilation are mentioned as three important phenomena. Finally, curvature of spacetime is presented: Gravity is not a force, but a geometric phenomenon that is connected to the curvature of spacetime.

Interestingly, when it comes to curvature of spacetime, the foci of the two Norwegian textbooks differ to a great extent. While Callin et al. only mention the geometric nature of gravity on the final page of the chapter on GR without detailed explanations [44], Jerstad et al. choose this topic as a main focus and present Einstein's struggle to find a mathematical description of GR from a historical point of view [45]. More in line with this second presentation, the Austrian textbook discusses curved spacetime in detail, but highlights features of non-Euclidian geometry instead of a historical approach. This difference in presentation of curved spacetime suggests that physics educators have not yet reached consensus on how to teach this feature of GR successfully on a qualitative level and an educational reconstruction of GR should take this aspect into account in particular.

To complement upper secondary interpretations of GR with the understanding of experts in the field, we studied an acclaimed university textbook in addition [48]. Here, the main emphasis is clearly on the geometry of spacetime as stated already in the introduction:

General relativity is Einstein's theory of space, time, and gravitation. (...) The essential idea is perfectly straightforward: while most forces of nature are represented by fields defined on spacetime, gravity is inherent in spacetime itself. In particular, what we experience as "gravitation" is a manifestation of the curvature of spacetime [48].

On the same line, the renowned physicist John Archibald Wheeler summarized GR with the famous phrase "Spacetime tells matter how to move; matter tells spacetime how to curve" [49]. Once more, we see that the challenge of teaching the physics of curved spacetime is crucial, since the explanation of gravity as a manifestation of curved spacetime is an important feature of GR according to leading experts.

Summarizing the findings from our analysis of textbooks, we divide the main features of GR into two categories: Key ideas that make up the conceptual foundation of GR and key phenomena that can be derived from this conceptual foundation. Our classification of the science content structure is summarized in Table I.

\section{B. MER-component 2: Student perspectives on general relativity according to research literature}

Aiming for a comprehensive understanding of students' perspectives and challenges when faced with GR, we analyzed the science education literature on GR. Surprisingly, there are hardly any systematic reviews on teaching GR and field-tested educational material is rare. Moreover, also studies on conceptual understanding of learners are scarce, leading Velentzas and Halkia in their study on the use of relativistic thought experiments to conclude that 
TABLE I. Science content structure: Key features of general relativity.

Conceptual foundation

Relativistic phenomena
The principle of equivalence:

- In small enough regions of spacetime, the laws of physics reduce to those of special relativity. In particular, this implies that locally there is no way to distinguish between the effect of a gravitational field and of being in a uniformly accelerated reference frame.

Reference frames and the principle of relativity:

- One can only define inertial frames locally.

- An inertial system is defined as a system in free fall.

- All reference frames are equivalent to formulate the laws of physics.

Spacetime and curvature:

- Gravity is not a force, but a manifestation of the curvature of spacetime.

- Energy and momentum influence spacetime to create curvature.

- Free particles move in straight paths in curved spacetime.

Gravitational bending of light:

- Massive objects deflect light.

Gravitational red shift:

- The frequency of light is influenced by gravity.

- Light that moves away from a massive body is redshifted.

Gravitational time dilation:

- A gravitational field influences the rate at which time passes.

- The farther a clock is away from a source of gravitation, the faster the time passes.
(...) the way physics theories of the twentieth century are taught in the classrooms has not yet been fully investigated by the educational community. Looking in the recent literature, the relative works are very few and the proposals are even fewer [7].

Indeed, out of more than 2000 articles on conceptual understanding in physics that were published within a period of 30 years, only eight focus on relativity while zero of them address GR explicitly [50]. Moreover, recent works on teaching relativity to preuniversity students focus usually on special as opposed to general relativity [8-10] or contribute to an important broader discussion on interpretations of GR [51] without suggesting actual learning goals that could guide the development of learning resources. Most of the publications that investigate learners' perspectives on GR study undergraduate instead of upper secondary students [11-13], an exception being Pitts et al. [1] and Kaur et al. [52] from the EinsteinFirst project [53]. The Einstein-First project aims to change the paradigm of school science teaching through the introduction of modern Einsteinian concepts of space and time, gravity and quanta at an early age. Through several so-called enrichment programs with Australian secondary school students, Einstein-First researchers found that already children of age 10-16 can understand basic principles of GR and are motivated by concepts of Einsteinian physics [1,52].

Teaching GR is challenging both technically and conceptually, because of the advanced math and the large amount of previous knowledge, notably special relativity, required [2,54,55]. Learners lack experience with relativistic phenomena and the counterintuitive nature of these phenomena poses yet another difficulty $[15,56]$. The disagreement between relativistic concepts and preexisting ideas of space and time that stem from everyday knowledge and classical physics teaching can lead to a situation where secondary school students suffer a "cognitive conflict" [7]. This conflict often manifests itself in upper secondary students not accepting consequences of the theory. For example, preexisting ideas on the absolute nature of time proved to be strong and students struggled to accept that time is different for moving observers, even though their own reasoning had led them to this conclusion [7].

Also, undergraduate students struggle with deeply rooted classical views on time and space. They have problems distinguishing between different reference frames and observers [11] which can hinder a proper understanding of the principle of equivalence. Indeed, in their study on alternative conceptions of the principle of equivalence, Bandyopadhyay and Kumar [12] identified an altered view of reference frames as "the most important cognitive transition that needs to be affected in the context of the principle of equivalence," [12]. Dimitriadi and Halkia [9] and Levrini and diSessa [10] confirmed that also upper secondary students have misleading conceptions of frames of reference and particularly the role of observers.

The most comprehensive study on conceptual difficulties in GR was conducted by Bandyopadhyay and Kumar [13]. They followed senior undergraduate students throughout a course on GR and investigated alternative conceptions of eight key issues that Einstein brought up in his own exposure of the subject [57]. In probing several aspects of GR, Bandyopadhyay and Kumar affirmed an 
TABLE II. Students' learning challenges in GR according to the literature.

$\begin{array}{ll}\text { General challenges } & \text { Specific challenges }\end{array}$

GR builds on an advanced level of mathematics.

GR requires a lot of background knowledge, in particular special relativity.

Students have no direct experience of relativistic phenomena.

The nature of relativistic phenomena seems to be counterintuitive to learners.

Preexisting ideas stemming from classical physics hinder understanding of GR.

Students struggle to accept the implications of

GR even when they have understood the

basic principles of the theory.
Students struggle with the definition of reference and inertial frames.

Students struggle with the role of observers in different reference frames.

Students struggle to apply the principle of equivalence.

Students cannot connect the equality of inertial and gravitational mass to the principle of equivalence and generally fail to see the difference between inertial and gravitational mass.

Students take the Euclidean nature of our universe for granted. assertion made by Sexl [58] that students often understand single ideas of GR but are not able to put these pieces together to understand the bigger picture. Moreover, they confirmed the above-mentioned difficulties and observed a lack of understanding for Einstein's achievement to explain the equality of inertial and gravitational mass. Students tended to think of Euclidean geometry as an obvious feature of space and often preferred an outside view on the universe as if it was embedded in a higher dimensional space. It is interesting to note that Bandyopadhyay and Kumar are the only authors who have reported on students' understanding of spacetime. However, their study only touches upon the nonEuclidean nature of spacetime and does not investigate student understanding of curved spacetime in detail which

\section{Einstein's happiest thought}

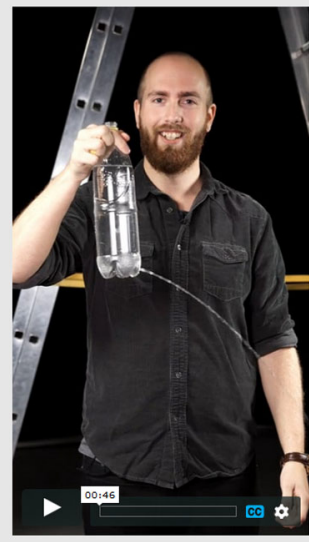

\section{Discuss in pairs}

Watch the film. What will happen to the jet of Water if he lets $g 0$ off the bottle? Will the water each other. Do not continue before you have discussed. You
will see the solution if you continue now.

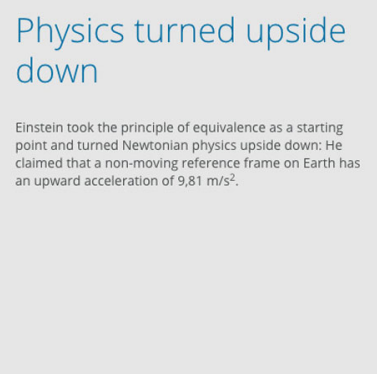

FIG. 3. A series of illustrations and thought experiments contrasts Newton's and Einstein's definition of an accelerated system to illustrate the concept of reference frames. (DH2, DH3).

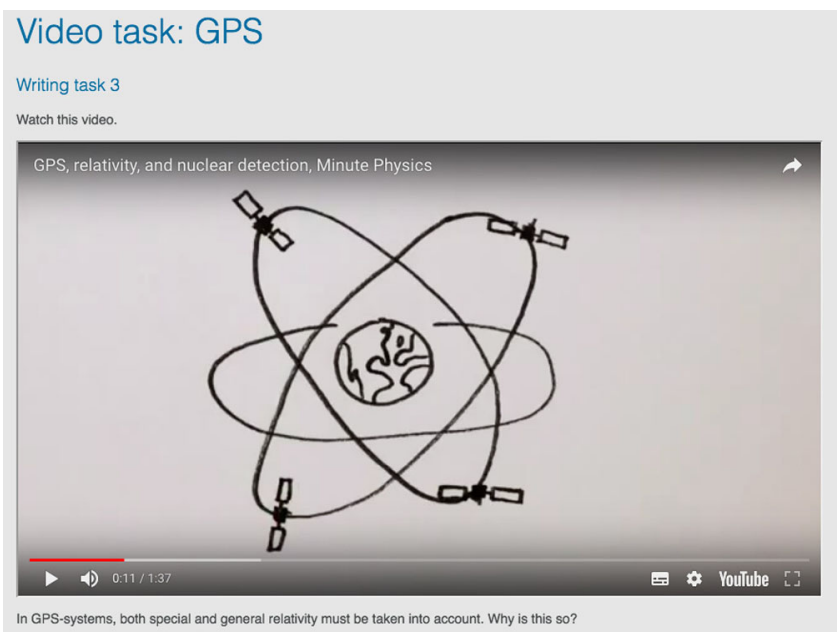

FIG. 4. A video exercise on GPS technology links the relativistic concept of time dilation to students' everyday life and allows them to make connections between special and general relativity. (DH4, DH5).

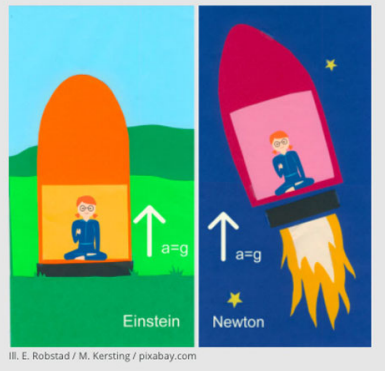

FIG. 2. A video exercise introduces students to the principle of equivalence and makes a connection to students' everyday life by asking them to discuss the physics of a falling water bottle. (DH2, DH5, DH7).

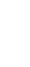
. 


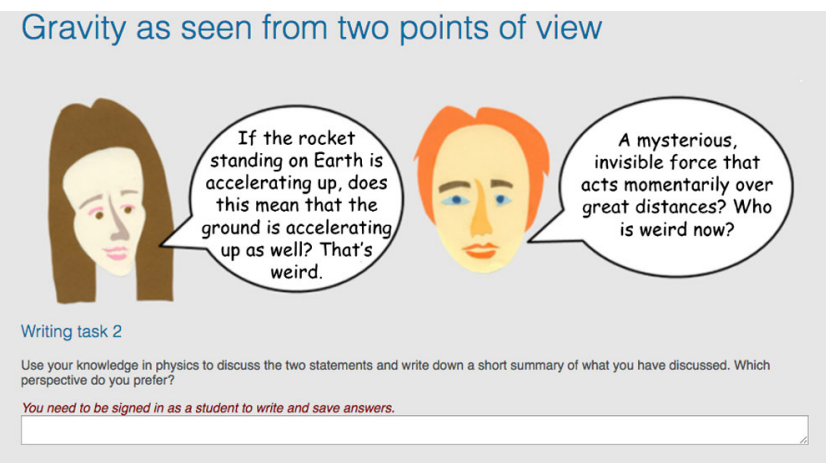

FIG. 5. We invite students to "talk physics" by discussing key concepts of GR. In this exercise, students are asked to discuss the two different viewpoints on gravity as held by Newton and Einstein. (DH7).

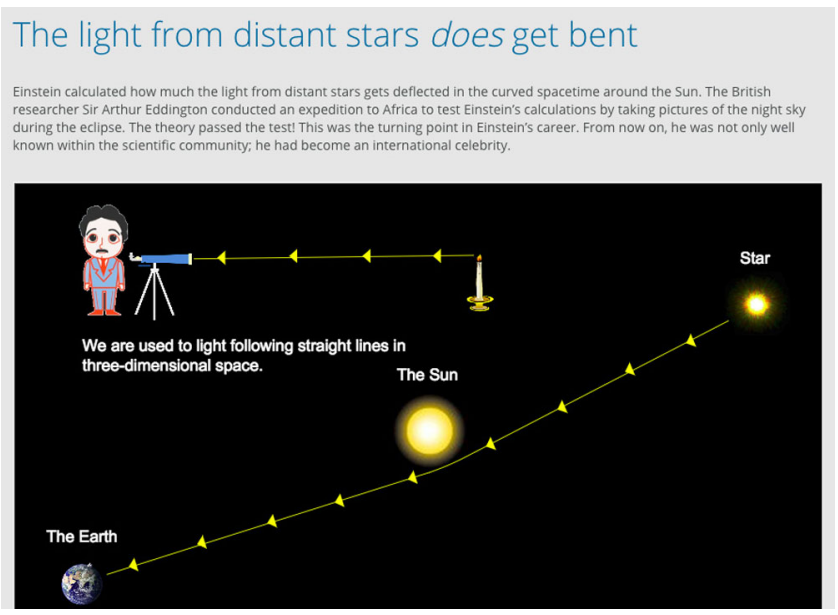

FIG. 6. An animated thought experiment presents gravitational bending of light as a phenomenon that GR predicts but that classical physics cannot explain. (DH2, DH3, DH5, DH8).

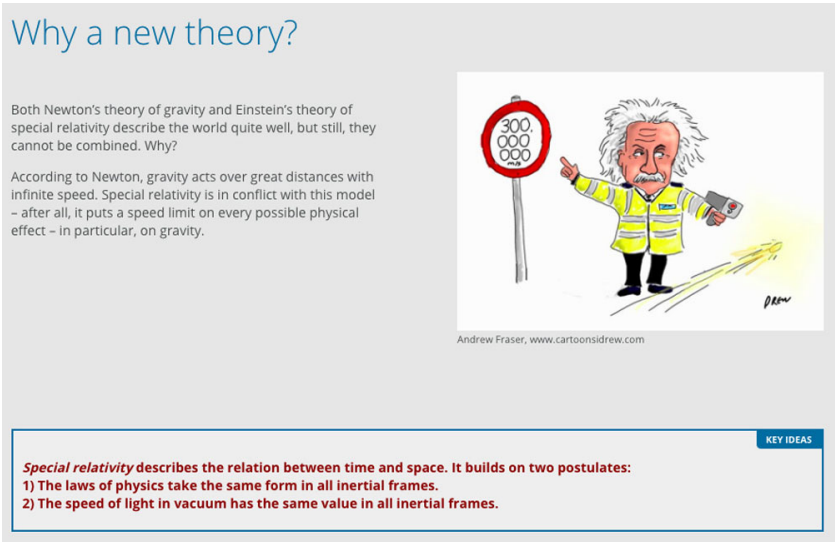

FIG. 7. The learning environment builds on students' previous knowledge of classical physics and special relativity to show the need for a new theory of gravity. (DH3, DH4).
Gravitational bending of light observed

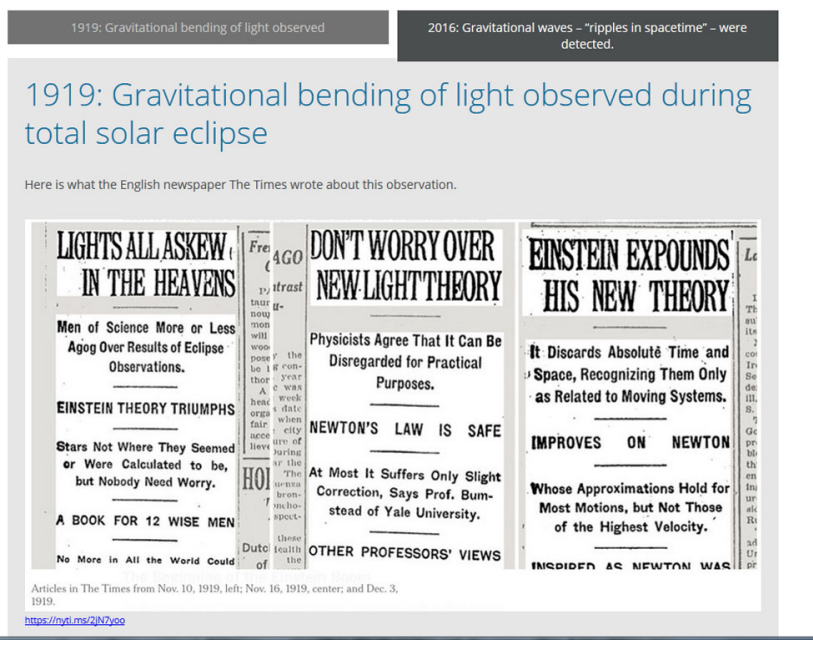

FIG. 8. To show that GR is a topic with historic and present-day relevance, we constrast the first experimental confirmation of GR to the recent breakthrough in gravitational wave astronomy. Students can move between newspaper headlines from 1919 and 2016. (DH5, DH6, DH8).

\section{Geometry in curved spaces}

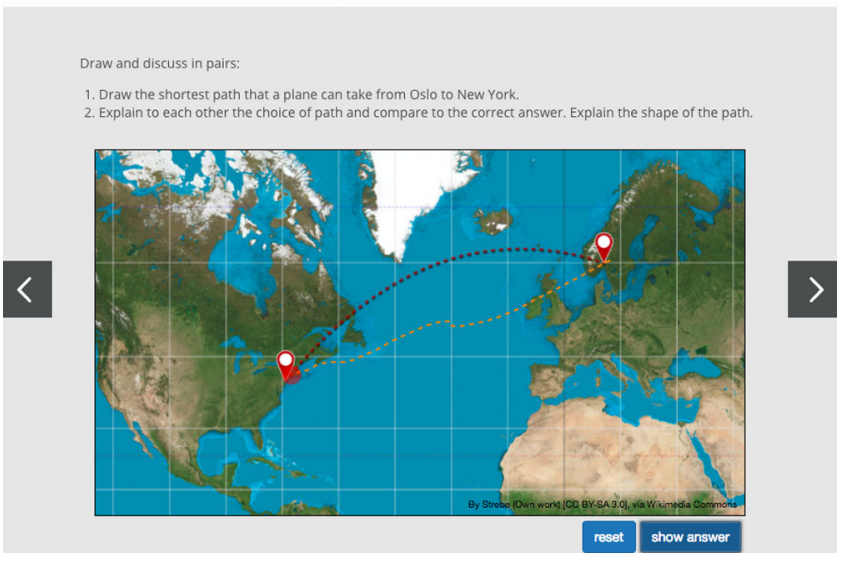

FIG. 9. Several exercises ask students to discuss in pairs to foster understanding for relativistic concepts. In this task, a flight route on the world map illustrates the geometric nature of gravity. (DH1, DH7).

therefore still seems to be a mostly unexplored issue in science education research.

Also, other parameters can make learning GR difficult, such as a lack of time for teaching [55] or a lack of suitable learning activities [54]. Yet, despite various challenges, relativity seems to engage students to a high degree [59] and "most or all students show much interest, independent of age, of gender and of their general interest in physics" [56]. In Table II, we present the findings on students' challenges according to the literature more concisely in summary. 
Do you want to know more?

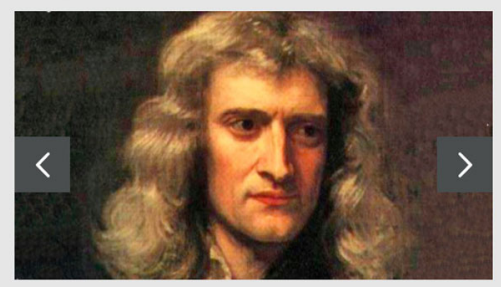

$$
\bullet \bullet \bullet \bullet
$$

FIG. 10. By presenting direct quotes of Newton and Einstein, we introduce students to the historical development of GR and present physics as a human endeavor. (DH8).

\section{MER-component 3: Development of the online learning environment}

We have identified key features of GR as they appear in textbooks and established an understanding of students' learning challenges through a literature search. We have argued for an instructional approach emphasizing students' use of language in the learning process and for including historical and philosophical perspectives. In this

section, we present how these findings and their mutual relationship have guided our transformation of the science content structure of GR into a content structure for instruction in accordance with the MER framework. This content structure of instruction lies at the heart of the design of our online learning environment.

Since a successful educational reconstruction of general relativity must take both content and design features into account, we suggest a twofold content structure that includes specific learning goals for GR in secondary physics supplemented with design hypotheses. Focusing on a qualitative presentation, we propose an educational reconstruction of GR based on the learning goals presented in Table III. To arrive at those learning goals, we have taken the key features of GR (Table I) and reformulated and structured them in a way that is suitable for teaching at the upper secondary level. The learning goals serve as an interpretation of the vague Norwegian curriculum goal and as a possible approach for other contexts where GR is to be presented to learners with some physics background, but without advanced mathematics. Our design hypotheses (Table IV) complement the learning goals to facilitate teaching and learning of GR. Figures 2-10 exemplify the implementation of the design hypotheses through screenshots of the online

TABLE III. GR content structure for instruction specified by learning goals.

Content $\quad$ Learning goals

Introduction

The principle of equivalence

Reference frames and principle of relativity

Relativistic phenomena (bending of light, gravitational redshift, time dilation)

Spacetime and curvature
Describe general relativity as a new theory of gravity.

Explain how the fact that Newton's force of gravity acts instantaneously contradicts Einstein's claim that nothing can move faster than the speed of light.

Use the fact that locally it is impossible to distinguish between a gravitational field and a uniform acceleration and/or between free fall and the absence of a gravitational field to explain how acceleration and gravity are equivalent phenomena.

Describe an inertial reference frame as a reference frame in free fall.

Explain that the laws of physics take the same form in all reference frames.

Give examples of phenomena that are predicted by GR but not by Newton's theory of gravity.

Describe how light travelling through the gravitational field of the sun is deflected and use the principle of equivalence to explain why this is predicted by GR.

Describe how time goes slower close to massive objects and use the principle of equivalence to explain why this is predicted by GR.

Explain how light that moves away from a gravitational source is redshifted and light that moves towards a gravitational source is blueshifted.

Explain how general relativity is a theory describing the relationship between space, time, and gravity.

Describe our universe as having three spatial and one temporal dimension.

Explain that gravity is not a force, but a geometric phenomenon.

Describe how mass curves spacetime and how curvature influences the movement of mass. 
TABLE IV. Design hypotheses for design of learning resources.

Design hypotheses

DH1 Students can grasp central ideas in GR qualitatively without advanced mathematics by relying on geometric ideas.

DH2 Thought experiments, analogies and visualizations of relativistic phenomena foster understanding in GR.

DH3 Emphasizing the break of relativistic with classical physics helps students to overcome their classical preconceptions.

DH4 Recalling background knowledge in special relativity allows students to align relativistic ideas from special relativity with general relativity.

DH5 Linking abstract topics to students' everyday life motivates and fosters understanding in GR.

DH6 Students are generally motivated by topics in relativistic physics, such as black holes and spacetime.

DH7 Use of language and talking physics facilitates understanding of abstract concepts in GR.

DH8 Students are interested in the historical development of GR and its philosophical implications.

learning environment. In Sec. VI we will critically discuss the design hypotheses in light of student experiences to arrive at finalized design principles according to the DBR framework.

\section{CLASSROOM IMPLEMENTATION AND METHODS}

\section{A. Implementation and data collection}

The design hypotheses, together with the learning goals, led us to develop an online learning environment designed for 180 min of classroom time, containing text and images, videos, animations and simulations, writing and discussion tasks, thought experiments, and contextualization using historical as well as everyday examples. The learning environment was implemented in six physics classrooms at four generally high-performing schools in the Oslo area in spring 2016. The students were in their final year of upper secondary school and enrolled in the most advanced physics course available in Norwegian upper secondary school, namely, Level 2 physics. The students' teachers, who had participated in the DBR process of developing and testing previous versions of the resources, led the physics lessons. Teaching comprised two units of 90 min each. In total, 122 upper secondary students (age 18-19), 44 girls (36\%) and 78 boys, and their six teachers participated. Informed consent to participate in the research was retrieved from all students and teachers.

To be able to follow students' learning paths, each lesson was observed by 1-2 field workers, one of whom was always the first author. In three classes, video recordings supplemented the observations. To investigate how students "talk physics," we asked students to make audio recordings of small-group discussions that are built into the learning environment. Students recorded the discussions with their smartphones and sent the files to the teacher, who forwarded them to researchers. Students' written responses were retrieved from the online learning platform. To gain insight into students' experiences with the learning environment and their own judgment of their learning in GR, we conducted four semistructured focus group interviews, one in each participating school with 5-8 participants per group and 25 students in total. The students were chosen by the teachers to allow for a balance of gender and to include both stronger and weaker students. To ensure consistency, the first author conducted all interviews. The interviews were based on an interview guide focusing on the design of the learning activities, use of language, and students' challenges and motivation.

\section{B. Analysis of data}

The first author and research assistants transcribed audio recordings from small-group discussions and focus group interviews, and these files, together with students' written responses, were imported into the Atlas.ti software for qualitative data analysis. Data sources for students' understanding of key concepts (RQ1) and for evaluation of the design hypotheses (RQ2) consist of focus groups and responses to written and audio exercises.

The analysis was based on thematic coding [60] and driven by our research questions. The first author performed the initial coding, and the coding process, formulation, and interpretation of findings, and the selection of representative quotes was discussed among ourselves over several rounds.

In the first stage of analysis, focus group interviews were coded for key concepts of GR that we chose as overarching themes (principle of equivalence, reference frames, spacetime, gravity, relativistic phenomena). In addition, focus group data were coded for features of our design hypotheses (language, history and philosophy, thought experiments, motivation, gap every-day, new way of thinking).

Written and oral responses were coded for correctness and for overarching themes (as above), which were again broken down into recurring motives that students drew on to support their arguments.

\section{RESULTS AND DISCUSSION}

To answer our research questions, we evaluated the participating students' understanding of key concepts in GR (RQ1) and investigated how students' experiences support or challenge the design hypotheses (RQ2). Concluding, we synthesized our empirical results to 
formulate design principles for the development of learning environments in GR for upper secondary school (RQ3). Specifically, our interest lay in evaluating the design hypotheses and understanding the process of learning in the setting where the activity and collaboration with peers and the teacher took place [61]. This focus aligns both with the sociocultural approach of ReleQuant, with the MER model, and with recent calls that invite educational researchers to "examine more closely how the meaning and functions of CSCL [Computer Supported Collaborative Learning] applications are actually constituted in practice" [62]. The authors translated quotes of students and teachers from Norwegian to English. We denote quotes from focus group interviews by FG, written responses by WR, and audio records of the oral discussions by OD.

\section{A. Student understanding of key features of GR}

Next, we present our findings on students' understanding of key concepts in GR.

\section{The principle of equivalence}

In the interviews, students could explain the principle of equivalence and were able to connect it to relativistic phenomena such as the deflection of light around the Sun. Nonetheless, some students found it difficult to believe that there is no experiment at all which could resolve whether one is accelerated or under the influence of gravity.

FG-student: No, for me, so it is mostly that it is a bit difficult to, sort of, believe that you cannot do any experiment to test whether you're not in an accelerated room instead of being in a pure gravitational field. This is something, which I think is a bit difficult to understand?

We introduced the principle of equivalence through a discussion exercise and an experiment with a leaking bottle of water that can be explained by classical physics and that relates to Einstein's own thought experiment (Fig. 2). This approach has been successful in connecting students' everyday experience to the physics of relativity. Students remembered the activity well and found it easier to relate to it than to the example of a falling or floating elevator that is usually found in textbooks and that has been analyzed in the literature before [7].

FG-student: I remember that there [in the online learning environment] were much easier examples to understand, because the book uses those examples that you yourself can't relate to. But in the project we used examples which we could actually feel familiar with. $F G$-researcher: Do you have some examples for that? FG-student: For example, the one, the water bottle.
However, many students would have liked a summarizing explanation at the end of the activity to connect the principle of equivalence explicitly to the physics of a falling water bottle. This criticism was partly confirmed by an analysis of the corresponding audio-recorded discussions. We found that the question "What will happen to the jet of water if the bottle falls?" was poorly formulated, because many students did not relate their discussion directly to the principle of equivalence but discussed related topics such as air resistance instead. Consequently, many groups did not come to the right conclusion, showing indeed the need for a clarifying summary as part of the activity. This finding suggests that learning activities in GR have to be very explicit when linking relativistic phenomena to students' everyday experience. As a consequence, questions for pair and group discussions have become more specific in the final learning environment.

\section{Reference frames}

In the interviews, none of the students mentioned the new definition of inertial frames explicitly, which could hint towards a lacking acknowledgement of the important reinterpretation of reference frames in GR [12]. The analysis of the audio discussions revealed that there seems to be a big gap between knowing the definition of an inertial system and being able to apply it to a given situation, suggesting that rote rather than deep learning had occurred. Students showed mixed understanding trying to find an answer to the question "According to GR, are you in an inertial reference frame while sitting in the classroom right now?" Even though many gave the correct answer, namely, that they are not in an inertial frame according to GR, the discussions revealed that students often did not feel confident in their reasoning. Students not trusting their own reasoning or sticking to classical views of accelerationeven though they could reproduce Einstein's definition of being accelerated-supports findings from Velentzas and Halkia [7].

OD—student 1: I thought we agreed on being in an inertial system.

OD-student 2: We are not...

OD-student 1: Yes, we are surely not in free fall, so then we are in an inertial system.

OD-student 2: Yes, we are influenced by acceleration. Never mind.

One group of students came to the conclusion that an inertial frame depends on the perspective of the observer and is therefore a relative concept; other students formulated similar thoughts but would not go so far as to say that one cannot give a definite answer to the question. Emphasizing that there exist several concepts in relativity that are not relative (the notion of inertial frames being one 
of them) might help students to connect novel ideas in GR to their previous knowledge of physics.

\section{Gravity and spacetime}

In an introductory exercise, students were asked to answer the question "What do you know about gravity?" The written responses revealed that there was great variation in the understanding of gravity. Ideas ranged from phenomenological to mathematical descriptions as well as personal experiences of gravity. To contrast students' initial knowledge with their ideas at the end of the unit, the nature of gravity was a topic in the focus group interviews. Most students answered in line with Einstein. However, they admitted that both Newton and Einstein seemed to have valid explanations, but Einstein's explanation was perceived as far away from real life.

FG-student 1: I think if you have to explain to an average person what gravity is, like just now, then it gets easier to say Newton's, yes, everything that has mass attracts mass and so, yes, then there is opposing force and attracts each other equally and stuff. But it, yes.

FG-student 2: Yes, I could have never gone and said I talk to mum about, about what Einstein meant, because she wouldn't have understood anything.

Connected to the idea of gravity is the notion of curved spacetime. In an activity at the end of the chapter on curved spacetime, students were asked to summarize Newton's and Einstein's description of gravity. The interviews revealed that spacetime is an engaging, yet challenging concept that the students felt very uncertain about:

FG-student: This is very exciting, because you can look at spacetime and then it curves if there is mass, around mass, but this is, you can see it in your mind, but then you think that you are in there, you are on the surface of the earth, you are sort of in a small sink. So then it gets sort of difficult to imagine it again, because if you take a look around you see the Universe, you don't think of being down in a well somehow; this is very difficult.

However, even though curved spacetime is perceived as a difficult concept, students generally found the geometric way of looking at space and time exciting. This interest supports a claim made by Levrini and Fantini [63], who argued that challenges in modern physics can be productive and engaging for upper secondary students.

This statement illustrates another finding that aligns with the perception of GR being far away from students' life: Students found it difficult to relate the abstract description of curvature to their own experience of gravity. While they could reproduce the general description that mass curves spacetime and the curvature of spacetime leads to gravitational effects, they struggled to explain why gravity keeps them grounded on the floor. This gap between abstract and experiential understanding of gravity is further elaborated on in a related publication from the ReleQuant project [64] and has also been identified among middle school students [1].

\section{Relativistic phenomena: Redshift, time dilation, and gravitational bending of light}

In the interviews, students could name gravitational bending of light and gravitational time dilation as relativistic phenomena that GR predicts. It seems that these phenomena have acted as a complement to the more abstract principles and have helped to bridge the gap between scientific concepts and students' everyday understanding of time, space, and gravity. Students exemplified movement through curved spacetime with light rays that bend around the sun. Moreover, they appreciated the historic perspective of introducing deflection of light in the context of the solar eclipse from 1919.

While the bending of light was well received by students, gravitational time dilation and redshift seemed to have been more problematic. Even though well remembered in the interviews, students found these features complicated. This observation was confirmed by an analysis of a written task in which the gravitational redshift of a light signal detected in a spaceship was used to explain time dilation. In their explanations, students often mixed up classical and relativistic concepts, presented incoherent arguments, or tried to argue with the relativity of observation to explain time dilation:

WR-student: The blueshift is relative to the rear, not to the space around the spaceship. This way, a detector in the spaceship will read different values for the number of wave crests than a detector in the surrounding space. This suggests the contraction of objects that accelerate.

Thus, when presenting applications of GR it is important to take enough time to explain the relativistic phenomena in detail instead of just using them as an example of a more general principle of the theory.

\section{B. Evaluation of design hypotheses for the GR learning environment}

The design of the learning environment was framed by eight design hypotheses. In this section, we will discuss these hypotheses in light of our empirical findings.

DH1: Students can grasp central ideas in GR qualitatively without advanced mathematics by relying on geometric ideas.

Using geometric ideas to foster understanding of the curvature of spacetime is a strategy that has been proposed 
before by education researchers and physicists [2,53, $54,65]$. Even though GR relies on an advanced mathematical foundation, the theory is geometric in its nature. We made use of this feature by developing learning activities that relied on geometry to explain curved spacetime. In the learning environment students encountered a simulation that presented Einstein's quest to find a geometric description of GR. Students were asked to discuss the form of a flight route on a world map to become familiar with the concept of shortest paths on curved surfaces (Fig. 9). The simulation continued to compare the geometry of the world map to the geometry of spacetime. We found that this geometric approach was very successful in engaging students and facilitated learning of the geometric features of curved spaces.

OD-student 1: We believe that eh...you don't fly along a straight line because the Earth is round, and...

OD-student 2: And then the shortest path becomes such a curved line instead of a straight line because the Earth is not a plane, like on a sheet. This is a bit exciting because then you know that maybe not always a straight line is the shortest path between two points.

However, it is not clear whether such a geometric approach actually did foster a deeper understanding for curvature in four-dimensional spacetime or whether it only helped students to grasp ideas of 2D and 3D spatial curvature. In another study, we looked closer at students' understanding of the space and time dimensions in GR exemplified by the rubber sheet analogy [66]. This analogy compares spacetime to a rubber sheet that gets distorted by massive objects. It is a popular, yet controversial way of illustrating GR $[2,53,67]$. We found that even though most students showed awareness of the limitations of an analogy that reduces the number of dimensions, many did not address the temporal dimension of spacetime explicitly [66].

DH2: Thought experiments, analogies and visualizations of relativistic phenomena foster understanding in $G R$.

Students lack direct experience of relativistic phenomena and one can use several strategies to deal with that problem. Generally, it seems that thought experiments have helped to deepen students' engagement with key concepts in GR. When asked about the role of thought experiments in the learning environment, students answered that those were engaging and challenged their understanding, thus making it easier to probe their own knowledge in GR.

FG-student: I actually liked all the thought experiments, because you have to, sort of, think a bit, now we have learned something and then you have to think a bit about why it is like this, how can you use this after all? Ehm, this was actually pretty nice, since then you are in a sort of different mode, you have to think and not only read something.

These findings support Velentza's and Halkia's [7] conclusion that thought experiments are useful educational tools to help students deal with abstract concepts in relativity. Thought experiments were especially successful to explain the principle of equivalence. Many students confirmed that the thought experiments of Einstein stepping off a tall building and of a laboratory in an accelerated spaceship helped them in their understanding.

The use of analogies is a more ambiguous issue. We observed a heavy dependence on the rubber sheet analogy when students talked about curved spacetime. The fact that this analogy was ubiquitous throughout the data set suggests that learners have a great need for visualizations. In particular, the analogy of the rubber sheet seems to inform the way students think about spacetime:

$F G$-researcher: What is gravity?

FG-student 1: Yes, I have this (laughs) warps in, eh, or according to Einstein warps, in, eh, curvature of space. Then, so like, this is sort of pretty difficult to explain that you need these visual, yes, you need it visualized to just understand it better.

FG-student 2: Like he said with this trampoline that, eh ...

FG-student 1: Yes, if you think of spacetime then it gets a lot easier to understand.

Students' reliance on the rubber sheet analogy could be partly explained by a lack of other convenient and visual ways to grasp the abstract idea, especially because a mathematical description is not accessible. These findings confirm the need for suitable visualizations as expressed by Kraus [56] and Weiskopf et al. [68].

However, the analogy troubled some students and they criticized the comparison, because it relied on gravity to explain gravity, which is a common criticism among physicists as well [67].

FG-student: Hm, the only problem I have with the rubber sheet analogy is that it requires a gravity (laughs) to make the model work as well. This is what I struggle with, because there won't be any curvature in space without a gravity that makes a well in it.

The use of this particular analogy should therefore be reconsidered in light of analogies being "two-edged swords" when viewed as educational tools [69]. Insufficient discussion of the flaws of the rubber sheet analogy in the learning environment might have led to unnecessary misconceptions. Teachers should therefore point out explicitly in which ways the rubber sheet analogy 
oversimplifies the geometric description of GR. This observation guided a revision of the learning environment that includes a new discussion task on the shortcomings of the rubber sheet analogy in its final version.

DH3: Emphasizing the break of relativistic with classical physics helps students to overcome their classical preconceptions.

Since preexisting ideas that stem from classical physics often hinder students' understanding, one can present the break between classical physics and GR explicitly to make students become aware of their own preconceptions. In the interviews, students repeatedly expressed the insight that GR greatly differs from classical physics. Often, they articulated the need for a new way of thinking to understand GR. Students found this shift of perspective challenging, but also exciting and fun:

FG-student: Otherwise, it was fun to just see everything from a completely different angle all the time, a different perspective, like ohh, you can look at this also in this way.

This finding supports our hypotheses that emphasizing the break between GR and classical physics can engage students and help them become aware of their classical understanding of physics. Moreover, such a strategy has already been successfully used in special relativity [7] and quantum physics [17].

DH4: Recalling background knowledge in special relativity allows students to align relativistic ideas from special with general relativity.

The interviews confirmed previous findings that students find special relativity with its new way of describing time and space difficult [9]. Moreover, we found that students tended to confuse principles of special and general relativity:

FG-student 1: Mass curves time and space, this was a bit difficult; I didn't manage to (mumble). And it is very weird to think that centimeter and meter are kind of different in movement than if they stand still. This I think is difficult to think, difficult to accept, or how, kind of, that if one centimeter and one meter are like. Yes, I think this was difficult.

$F G$-student 2: This was special relativity.

FG-student 1: I have little overview on that. But I think this was difficult to understand.

These findings suggest that one way of helping students overcome their confusion might be to present more clearly the distinction between key concepts of special and general relativity and how GR aligns with special relativity and classical physics. Because of time and curriculum constraints in schools, drawing on special relativistic concepts could make it even more difficult for students to understand abstract concepts in GR. In particular, the concept of reference frames requires students to handle conflicting definitions in classical physics, special relativity, and general relativity.

DH5: Linking abstract topics to students' everyday life motivates and fosters understanding in GR.

Learners experience the nature of relativistic phenomena as counterintuitive. Overall, using everyday examples to illustrate relativistic ideas worked well to explain the principle of equivalence, the geometry of curved surfaces and relativistic phenomena. As explained in Sec. VI.A and in Sec. VI.B in DH1, both the discussion exercise about curved flight routes on flat maps (Fig. 9) and the physics of a falling bottle of water (Fig. 2) stimulated discussions among students and were mentioned in the interviews. Students understood the application of GR in GPS technology well. In an exercise in which students were asked to explain how special and general relativity affect GPS systems (Fig. 4), almost all students gave a correct or partly correct explanation and many supplemented their responses with detailed explanations showing that students were able to connect newly learned content in GR to their previous knowledge:

WR-student: If you look at special relativity, then moving clocks will go a bit slower than clocks at rest. You have to take this into account considering that the satellites, and the Earth, follow their orbit with a certain velocity. But, if you look at general relativity, clocks that are in a gravitational field go slower than clocks that are outside of a gravitational field. This means that the clocks on Earth go slower than the clocks in the GPS satellites, so you have to take this into account as well.

Thus, emphasizing links between everyday life and abstract phenomena seems to be fruitful when introducing students to GR. This observation aligns with a similar one in quantum physics [17].

DH6: Students are generally motivated by topics in relativistic physics, such as black holes and curved spacetime.

Concerning their motivation and attitude towards learning GR, students confirmed our hypothesis, in line with observations of Zahn and Kraus [2]. They wanted to learn more about the nature of space and time because they perceived the topic to be modern and relevant:

FG-student 1: ...the more I think about it, the more fascinated do I get...

FG-student 2: ... what we have learned has been very old-old knowledge—so (...) 
TABLE V. Empirically based design principles for learning resources in GR.

Basic principles and specific features

1. Emphasize how GR relates to and sometimes breaks with classical physics.

(a) Present the need for a new theory of gravity by showing that special relativity and classical mechanics are irreconcilable.

(b) Present relativistic phenomena such as gravitational bending of light and time dilation to show that GR extends the scope of classical physics.

(c) Point out how the definition of inertial frames in GR differs from similar notions in special relativity and classical mechanics. Ask students to apply the abstract definition of an inertial frame to specific problems.

(d) Show that there exist concepts in GR that are NOT relative, such as the notion of inertial reference frames, to help students connect relativistic ideas to their classical understanding of physics.

2. Link key concepts of GR to students' life worlds to counteract the lack of experience with relativistic phenomena.

(a) Use everyday examples to illustrate relativistic ideas and to enable students to connect GR to their everyday life. GPS technology can exemplify gravitational time dilation and the geometry of world maps can illustrate motion in curved spaces.

(b) Use thought experiments as educational tools to help students understand abstract concepts in GR. Thought experiments that illustrate free fall and weightlessness are particularly successful when explaining the principle of equivalence.

(c) Use analogies with caution. State shortcomings of analogies explicitly to prevent the formation of misconceptions. In particular, explain how the rubber sheet analogy oversimplifies the notion of curved spacetime.

(d) Use visualizations in the form of digital simulations and animations to introduce students to relativistic concepts and to prevent the formation of misconceptions.

3. Draw on students' prevailing motivation and interest to introduce key concepts in GR.

(a) Use astronomical phenomena to engage students. Gravitational lensing around black holes can illustrate gravitational bending of light and curvature of spacetime. Thought experiments involving spaceships can illustrate the principle of equivalence.

(b) Present GR in light of its historical development. The solar eclipse in 1919 can serve as historical example for an experimental verification of GR. Relate Einstein's quest to find a new theory of gravity to abstract descriptions of GR.

(c) Emphasize epistemological aspects of GR and explain how Einstein's new interpretation of space, time, and gravity has shaped our worldview.

(d) Present GR as an active field of research by referring to the recent observation of gravitational waves.

4. Invite students to use written and oral language to facilitate understanding of abstract concepts in GR.

(a) Give students the opportunity to "talk physic"" with their peers by using discussion tasks that probe conceptual understanding of key concepts in GR.

(b) Ask students to summarize their understanding of key concepts in written exercises to let them practice the use of new physics vocabulary.

(c) Use plenary discussions guided by the teacher to consolidate understanding of GR and resolve misconceptions.

(d) Explain that our qualitative understanding of GR can be made rigorous by employing advanced mathematics.

when we learned about those gravity waves (...) I found it very interesting.

Students' fascination might be due to popular culture, because GR has become part of the 20th century cultural heritage [1,7] and Einstein has become a scientific icon. The recent detection of gravitational waves [5] can have contributed to this perspective, since many students mentioned the discovery.

\section{DH7: Use of language and talking physics facilitates understanding of abstract concepts in GR.}

Generally, the focus on using language to build and articulate physics understanding and insight was well received by students. The focus groups interviews supported previous findings on using language in science classrooms [17,43,70]: Talking physics fostered students' overall understanding in GR and discussions with peers and with the teacher were experienced as an engaging variation from regular teaching. In particular, students appreciated to think aloud, and they liked that their understanding of GR was challenged by discussions, which forced them to reason and to find arguments:

FG-student: I understand it a lot better if I have to explain it, so even though I haven't quite understood it myself, I start to try to explain it. So this, sort of, the pieces fit together while I work on that, so this helps. This way I get a much clearer picture of how this is.

Nonetheless, students asked for more mathematical approaches and easy calculations to probe their understanding as well. This was a recurrent critique in the interviews that relates to the frustration that students experience when approaching GR only qualitatively. This finding shows that students are aware of the close relationship between mathematics and physics [71]. However, the challenge in GR is that we do not have easy calculations at hand and that students have to rely almost exclusively on nonmathematical explanations:

FG-student: (...) it is difficult to understand like.

FG-researcher: Yes, but could we have made it easier to understand? 
FG-student: This is a bit difficult to say, but maybe like try to explain by doing a, a short math calculation (...) ehm, a bit more proof of it, it doesn't need to be a very complicated proof.

To counteract the formation of frustration among students, teachers have to raise awareness for different ways of learning physics: qualitative understanding and historical and philosophical perspectives should be seen as valuable learning goals in their own right, enabling students on preuniversity level to grasp important areas of modern physics without the full mathematical-formalistic "toolbox" [72].

\section{DH8: Students are interested in the historical develop-} ment of GR and its philosophical consequences.

Aside from aspects regarding the specific content of GR, students were particularly motivated by approaches relying on the history and philosophy of science. They approved of a historic perspective that gave them insight into the development of GR. Being able to follow Einstein's reasoning and his struggle to find a new theory of gravity seemed to enable them to draw connections to previous knowledge and to the theory as such. In particular, students appreciated the famous experimental verification of GR during the solar eclipse in 1919 (Fig. 8).

FG-student: I think it was very interesting that we kind of learned about, like, the history as well kind of. How this developed and this, (...) experiment with when they test bending of light with him, I don't remember his name, Sir...

FG-researcher: Sir Eddington.

$F G$-student: Yes, Eddington, that someone tested this kind of and verified it.

This supports previous research [73] highlighting how historical examples may support motivation and learning in physics.

\section{Empirically based design principles for developing GR learning resources}

After synthesizing our findings from the classroom trials with our design hypotheses and with previously published research, we now formulate design principles (RQ3) that we believe can guide the design of learning resources in GR and that foster qualitative understanding and encourage collaborative learning. The empirically based design principles follow a two-level structure with four basic principles that are specified by more specific features for each of them, as shown in Table V. These principles together with the insight on students' challenges identified in this study informed revisions of our learning environment. The revisions led to the final version that is now freely accessible on the Norwegian learning platform Viten [74].

\section{SUMMARY AND CONCLUSION}

The aim of this study was to describe and propose an educational reconstruction of GR for upper secondary physics students. Following the framework of MER, we identified key features of GR and studied learners' difficulties based on an analysis of physics textbooks and relevant literature. These results enabled us to turn the science content structure of GR into a content structure for instruction. DBR methods guided the development and evaluation of a collaborative online learning environment which were based on a sociocultural view of learning and a historical-philosophical approach to teaching GR. By extracting central principles for the design of an online learning environment in GR and by characterizing students' understanding and learning challenges, we added new empirical results to two of the three components in the MER framework. Seeing that field-tested educational material in GR is still rare, this study thus contributes to a growing body of research into teaching and learning GR at secondary school level.

Our findings corroborate earlier results reported in the literature $[1,7,13,52]$ and have added to a deeper and more comprehensive understanding of students' challenges in learning GR. Specifically, we have presented first empirical results on students' understanding of curved spacetime, which is scarce in the literature. In summary, our findings indicate that upper secondary students can obtain a qualitative understanding of GR when provided with appropriately designed learning resources and sufficient scaffolding of learning through interaction with teachers and peers.

By synthesizing our empirical findings with the design hypotheses that we based on a sociocultural perspective and historical and philosophical approaches to learning physics, our final design principles are equally grounded in theory and practice. The principles arose from an iterative process of development and their formulation completes our proposed educational reconstruction of GR targeted at upper secondary students.

The online learning environment presented in this paper was developed to help Norwegian upper secondary students achieve the specific competence aims in the national curriculum for physics. Thus, design principles may need to be adapted to other groups of learners and different curricular aims. While our results are not generalizable per se, we claim, however, that our results demonstrate the feasibility of communicating central aspects of GR qualitatively, without mathematics, and that our design principles represent insights that may be quite broadly applicable.

We anticipate future research that will throw further light on how GR can be communicated to and understood by different groups of learners as well as research investigating students' learning processes in more detail. GR is an important pillar of modern physics. We believe that our take on an educational reconstruction of GR brings us one step closer to teaching 
students our most contemporary scientific understanding of the Universe, thus turning GR into an important pillar of modern physics education as well.

\section{ACKNOWLEDGMENTS}

We would like to thank the Norwegian Centre for Science Education for their support in developing the online learning environment in general relativity and for hosting it on the learning platform Viten. In particular, we would like to thank Øystein Sørborg for his skillful implementation of the learning environment and for his many ideas and comments that improved its overall quality. We also thank the participating students, teachers, and colleagues in the ReleQuant research group. This work was supported by the Research Council of Norway (Project No. 246723) and by the Olav Thon Foundation.
[1] M. Pitts, G. Venville, D. Blair, and M. Zadnik, An exploratory study to investigate the impact of an enrichment program on aspects of einsteinian physics on year 6 students, Res. Sci. Educ. 44, 363 (2014).

[2] C. Zahn and U. Kraus, Sector models-a toolkit for teaching general relativity. part 1: Curved spaces and spacetimes, Eur. J. Phys. 35, 055020 (2014).

[3] D. Blair, E. K. Henriksen, and M. Hendry, Why don't we teach Einstein's theories school?, The Conversation (2016), retrieved from: https://theconversation.com/whydont-we-teach-einsteins-theories-in-school-69991.

[4] N. Christensen and T. Moore, Teaching general relativity to undergraduates, Phys. Today 65, 41 (2012).

[5] B. P. Abbott et al., Observation of Gravitational Waves from a Binary Black Hole Merger, Phys. Rev. Lett. 116, 061102 (2016).

[6] M. Egdall, Teaching general relativity to the layperson, Phys. Teach. 47, 522 (2009).

[7] A. Velentzas and K. Halkia, The use of thought experiments in teaching physics to upper secondary-level students: Two examples from the theory of relativity, Int. J. Sci. Educ. 35, 3026 (2013).

[8] O. Levrini, The role of history and philosophy in research on teaching and learning of relativity in International Handbook of Research in History, Philosophy and Science Teaching, edited by M. R. Matthews (Springer, Dordrecht, 2014), pp. 157-181.

[9] K. Dimitriadi and K. Halkia, Secondary students' understanding of basic ideas of special relativity, Int. J. Sci. Educ. 34, 2565 (2012).

[10] O. Levrini and A. A. DiSessa, How students learn from multiple contexts and definitions: Proper time as a coordination class, Phys. Rev. ST Phys. Educ. Res. 4, 010107 (2008).

[11] D. Gousopoulos, E. Kapotis, and G. Kalkanis, Students' difficulties in understanding the basic principles of Relativity after standard instruction, in Electronic Proceedings of the ESERA 2015 Conference. Science education research: Engaging learners for a sustainable future, Part 1, edited by J. Lavonen, K. Juuti, J. Lampiselkä, A. Uitto, and K. Hahl (co-editors O. Finlayson and R. Pinto) (University of Helsinki, Helsinki, Finland, 2015), pp. 169-175, ISBN 978-951-51-1541-6.
[12] A. Bandyopadhyay and A. Kumar, Probing students' ideas of the principle of equivalence, Eur. J. Phys. 32, 139 (2010).

[13] A. Bandyopadhyay and A. Kumar, Probing students' understanding of some conceptual themes in general relativity, Phys. Rev. ST Phys. Educ. Res. 6, 020104 (2010).

[14] http://www.mn.uio.no/fysikk/english/research/projects/ relequant/index.html.

[15] E. K. Henriksen, B. Bungum, C. Angell, C. W. Tellefsen, T. Frågåt, and M. Vetleseter Bøe, Relativity, quantum physics and philosophy in the upper secondary curriculum: challenges, opportunities and proposed approaches, Phys. Educ. 49, 678 (2014).

[16] K. Krijtenburg-Lewerissa, H. J. Pol, A. Brinkman, and W. R. Van Joolingen, Insights into teaching quantum mechanics in secondary and lower undergraduate education: A literature review, Phys. Rev. Phys. Educ. Res. 13, 010109 (2017).

[17] B. Bungum, E. K. Henriksen, C. Angell, C. W. Tellefsen, and M. V. Bøe, ReleQuant-Improving teaching and learning in quantum physics through educational design research, Nord. Nord. Stud. Sci. Educ. 11, 153 (2015).

[18] L. Vygotsky, Thought and Language (MIT Press, Cambridge, MA, 1962).

[19] J. L. Lemke, Talking Science: Language, Learning, and Values (Ablex Publishing Corporation, Norwood, NJ, 1990).

[20] E. Mortimer and P. Scott, Meaning Making in Secondary Science Classrooms (Open University Press, Maidenhead and Philadelphia, 2003).

[21] F. Abd-El-Khalick, Teaching with and about nature of science, and science teacher knowledge domains., Sci. Educ. 22, 2087 (2013).

[22] M. Monk and J. Osborne, Placing the history and philosophy of science on the curriculum: A model for the development of pedagogy, Sci. Educ. 81, 405 (1997).

[23] R. Duit, H. Schecker, D. Höttecke, and H. Niedderer, Teaching physics, in Handb. Res. Sci. Educ., edited by N. G. Lederman and S. K. Abell (Routledge, New York, 2014), pp. 434-456.

[24] R. Renstrøm, Kvantefysikkens utvikling i fysikklærebøker, vitenskapshistorien og undervisning. [The Development of Quantum Physics-in Physics Textbooks, in the History of 
Science, and in the Classroom] (Doctoral dissertation), University of Oslo, 2011.

[25] The Norwegian Directorate for Education and Training, Physics - programme subject in programmes for specialization in general studies (2006), retrieved from: https:// www.udir.no/k106/FYS1-01/Hele/Kompetansemaal/ fysikk-2.

[26] R. Duit, H. Gropengießer, U. Kattmann, M. Komorek, and I. Parchmann, The model of educational reconstruction-a framework for improving teaching and learning science, in Science Education Research and Practice in Europe: Retrospective and Prospective, edited by D. Jorde and J. Dillon (Sense Publishers, Rotterdam, 2012), pp. 13-37.

[27] T. Anderson and J. Shattuck, Design-based research: A decade of progress in education research?, Educ. Res. 41, 16 (2012).

[28] M. Méheut and D. Psillos, Teaching-learning sequences: aims and tools for science education research, Int. J. Sci. Educ. 26, 515 (2004).

[29] M. Komorek and U. Kattmann, The model of educational reconstruction, in Four Decades of Reserach in Science Education-from Curriculum Development to Quality Improvement, edited by S. Mikelskis-Seifert, U. Ringelband, and M. Brückmann (Waxmann, Münster, 2008), pp. 171-191.

[30] R. Duit, M. Komorek, and J. Wilbers, Studies on educational reconstruction of chaos theory, Res. Sci. Educ. 27, 339 (1997).

[31] M. Komorek and R. Duit, The teaching experiment as a powerful method to develop and evaluate teaching and learning sequences in the domain of non-linear systems, Int. J. Sci. Educ. 26, 619 (2004).

[32] Electronic Proceedings of the ESERA 2015 Conference. Science education research: Engaging learners for a sustainable future, edited by J. Lavonen, K. Juuti, J. Lampiselkä, A. Uitto, and K. Hahl (University of Helsinki, Helsinki, Finland, 2016), ISBN 978-951-51-1541-6.

[33] A. Laherto, An analysis of the educational significance of nanoscience and nanotechnology in scientific and technological literacy, Sci. Educ. Int. 21, 160 (2010).

[34] A. Laherto, Informing the development of science exhibitions through educational research, Int. J. Sci. Educ. Part B 3, 121 (2013).

[35] K. Niebert and H. Gropengießer, The model of educational reconstruction: A framework for the design of theory-based content specific interventions. The example of climate change, in Educational design research-Part B: Illustrative cases, edited by T. Plomp and N. Nieveen (SLO, Enschede, the Netherlands, 2013), pp. 511-532.

[36] S. Reinfried, U. Aeschbacher, P. M. Kienzler, and S. Tempelmann, The model of educational reconstructiona powerful strategy to teach for conceptual development in physical geography: the case of water springs, Int. Res. Geogr. Environ. Educ. 24, 237 (2015).

[37] S. McKenney and T.C. Reeves, Conducting Educational Design Research, 1st ed. (Routledge, New York, 2012).

[38] W. Sandoval and P. Bell, Design-based research methods for studying learning in context, Educ. Psychol. 39, 199 (2004).

[39] D. C. Edelson, Design research: What we learn when we engage in design, J. Learn. Sci. 11, 105 (2002).
[40] A. Collins, D. Joseph, and K. Bielaczyc, Design research: Theoretical and methodological issues, J. Learn. Sci. 13, 15 (2004).

[41] J. G. Greeno, A. M. Collins, and L. B. Resnick, Cogn. Learning, in Handbook of Educational Psychology, edited by D. C. Berliner and R. C. Calfee (US: Macmillan Library Reference USA, New York, NY; Prentice Hall International, London, England, 1992), pp. 15-46.

[42] L. S. Vygotsky, Mind in Society: The Development of Higher Psychological Processes (Harvard University Press, Cambridge, MA, 1978).

[43] E. K. Henriksen and C. Angell, The role of "talking physics" in an undergraduate physics class using an electronic audience response system, Phys. Educ. 45, 278 (2010).

[44] N. P. Callin, J. Pålsgård, R. Stadsnes, and C. W. Tellefsen, ERGO Fysikk 2 (Aschehoug, Oslo, 2012).

[45] P. Jerstad, B. Sletbak, A. A. Grimenes, R. Renstrøm, O. B. Holm, and M. Nymo, RomStoffTid Fysikk 2 (Cappelen Damm, Oslo, 2014).

[46] Austrian Federal Ministry of Education, and Women's Affairs, Austrian High School Curriculum in Physics (2004).

[47] M. Apolin, Big Bang-Physik 8, 1st ed. (Österreichischer Bundesverlag Schulbuch GmbH \& Co. KG, Wien, 2008).

[48] S. M. Carroll, Spacetime and geometry, Spacetime and Geometry: An Introduction to General Relativity (Pearson, Chicago, 2003).

[49] J. A. Wheeler, Geons, Black Holes, and Quantum Foam: A Life in Physics (Norton, New York, 1998).

[50] R. Duit, Bibliography-STCSE (Students' and Teachers' Conceptions and Science Education) (Kiel, 2009), retrieved from: http://archiv.ipn.uni-kiel.de/stcse/.

[51] O. Levrini, Reconstructing the basic concepts of general relativity from an educational and cultural point of view, Sci. Educ. 11, 263 (2002).

[52] T. Kaur, D. Blair, J. Moschilla, W. Stannard, and M. Zadnik, Teaching Einsteinian physics at schools: Part 3, Review of research outcomes, Phys. Educ. 52, 065014 (2017).

[53] T. Kaur, D. Blair, J. Moschilla, W. Stannard, and M. Zadnik, Teaching Einsteinian physics at schools: Part 1, Models and analogies for relativity, Phys. Educ. 52, 065012 (2017).

[54] A. A. diSessa, An elementary formalism for general relativity, Am. J. Phys. 49, 401 (1981).

[55] R. M. Wald, Resource Letter TMGR-1: Teaching the mathematics of general relativity, Am. J. Phys. 74, 471 (2006).

[56] U. Kraus, First-person visualizations of the special and general theory of relativity, Eur. J. Phys. 29, 1 (2007).

[57] A. Einstein, Relativity: The Special and the General Theory (Bonanza Books, New York, 1961).

[58] R. U. Sexl, Relativitätstheorie als didaktische Herausforderung [Theory of relativity as didactical challenge], Naturwissenschaften 67, 209 (1980).

[59] C. Angell, Ø. Guttersrud, E. K. Henriksen, and A. Isnes, Physics: Frightful, but fun. Pupils' and teachers' views of physics and physics teaching, Sci. Educ. 88, 683 (2004).

[60] V. Braun and V. Clarke, Using thematic analysis in psychology, Qual. Res. Psychol. 3, 77 (2006).

[61] A. Kluge and S. M. Bakken, Simulation as science discovery: Ways of interactive meaning-making, Res. Pract. Technol. Enhanc. Learn. 05, 245 (2010). 
[62] H. C. Arnseth and S. Ludvigsen, Approaching institutional contexts: Systemic versus dialogic research in CSCL, Int. J. Comput. Collab. Learn. 1, 167 (2006).

[63] O. Levrini and P. Fantini, Encountering productive forms of complexity in learning modern physics, Sci. Educ. 22, 1895 (2013).

[64] R. Steier and M. Kersting, Metaimagining and embodied conceptions of spacetime (to be published).

[65] J. B. Hartle, General Relativity in the undergraduate physics curriculum, Am. J. Phys. 74, 14 (2006).

[66] M. Kersting and R. Steier, Understanding curved spacetime - the role of the rubber sheet analogy in learning general relativity (to be published).

[67] R. R. Gould, Why does a ball fall?: A new visualization for Einstein's model of gravity, Am. J. Phys. 84, 396 (2016).

[68] D. Weiskopf et al., Explanatory and illustrative visualization of special and general relativity, IEEE Trans. Visual. Comput. Graph. 12, 522 (2006).
[69] A. G. Harrison and D. F. Treagust, Teaching and Learning with Analogies: Friend or Foe in Metaphor and Analogy in Science Education, edited by A. G. Harrison and S. M. Ritchie (Springer, Netherlands, 2006), pp. 11-24.

[70] Y.-C. Chen, S. Park, and B. Hand, Examining the use of talk and writing for students' development of scientific conceptual knowledge through constructing and critiquing arguments, Cognit. Instr. 34, 100 (2016).

[71] O. Uhden, R. Karam, M. Pietrocola, and G. Pospiech, Modelling mathematical reasoning in physics education, Sci. Educ. 21, 485 (2012).

[72] M. V. Bøe, E. K. Henriksen, and C. Angell, Actual versus implied physics students: How students from traditional physics classrooms related to an innovative approach to quantum physics, Sci. Educ. (2018).

[73] M. Chandler, Philosophy of gravity: Intuitions of fourdimensional curved spacetime, Sci. Educ. 3, 155 (1994).

[74] www.viten.no/relativity. 